in einer später im gleichen Sinne ergangenen Entscheidung zur Verwendung der Bezeichnung „,Spezialist für Verkehrsrecht" im Briefkopf eines Rechtsanwalts formuliert: „Die durch die werberechtlichen Regelungen geschützten Rechtsgüter und insoweit vom Normgeber verfolgten Ziele rechtfertigen es nicht, Angaben und Zusätze, die eine ausgeübte Tätigkeit näher charakterisieren sollen, ohne Rücksicht auf ihren Sinn und Zweck oder ihren Informationswert für Dritte zu verbieten."2 Beizupflichten ist dem von Kleine-Cosack hierzu gegebenen Hinweis, wonach es in so gelagerten Fällen nur noch um die Frage geht, wann im Einzelfall bei einer Werbung mit entsprechenden Begriffen eine Irreführung i. S. des $₫ 5 \mathrm{UWG}$ gegeben ist ${ }^{3}$. Ein solches Vorgehen liegt voll im Trend der europäischen Rechtsentwicklung, wonach das Werberecht der freien Berufe zunehmend an Bedeutung verliert zugunsten des allgemeinen Wettbewerbsrechts ${ }^{4}$.

Mit Recht sieht das Landesberufsgericht entgegen der Antragstellerin in der Verwendung des Begriffs ,Nasenchirurg" keine Verwechslungsgefahr, auch nicht im Hinblick darauf, dass der Beschuldigte in den beanstandeten Anzeigen nicht zugleich seine Facharztbezeichnung führt. \5 UWG verbietet irreführende Angaben, nicht aber deshalb auch unvollständige Angaben. Die Angaben müssen nur wahr sein, eine Pflicht zur Vollständigkeit besteht nicht. Eine Verpflichtung, bereits in Werbeanzeigen negative Eigenschaften des eigenen Angebots offenzulegen, besteht nur insoweit, als dies zum Schutz der Werbeadressaten unerlässlich ist ${ }^{5}$.

Im vorliegenden Fall bietet der Sachverhalt keinen sachlich begründeten Anlass zu Zweifeln am Wahrheitsgehalt der Angaben bei den hier in Rede stehenden Eintragungen. Denn nach der Weiterbildungsordnung und den Richtlinien über den Inhalt der Weiterbildung sind Voraussetzung für die Anerkennung als Facharzt für HNO-
Heilkunde u.a. der Nachweis von 25 (Anhaltszahl) operativen Eingriffen an der Nase, plastischen Maßnahmen geringeren Schwierigkeitsgrades an der Nase sowie die Mitwirkung bei Eingriffen höherer Schwierigkeitsgrade, u.a.z.B. bei neuroplastischen Eingriffen (vgl. Ziff. 8.1 der Weiterbildungsordnung Baden-Württemberg, Stand: 1.10.2009).

Sonach erfordert die Anerkennung als Facharzt für HalsNasen-Ohrenheilkunde u.a. den Nachweis von Kenntnissen und Erfahrungen gerade auch im Bereich des speziellen Leistungsangebots des Beschuldigten.

Unter diesen Umständen bestand für den Beschuldigten keinerlei Anlass für die Aufnahme seiner Facharztbezeichnung in die hier streitigen Veröffentlichungen. Damit wird auch der selbstverständlichen Forderung des BVerfG Rechnung getragen, dass ein Arzt, der mit Bezeichnungen wirbt, die das Weiterbildungsrecht nicht kennt, jedenfalls über besondere, von der Ärztekammer jederzeit überprüfbare Erfahrungen in den betreffenden Bereichen verfügen müsse ${ }^{6}$. Diese Uberprüfung fällt den Kammern bei HNO-Ärzten leichter als bei Allgemeinchirurgen, zu deren Pflichtweiterbildung operative Eingriffe speziell an der Nase nicht gehören. Mangels gegenteiliger Anhaltspunkte kann die Ärztekammer im vorliegenden Fall davon ausgehen, dass der Beschuldigte die für nasenchirurgische Eingriffe erforderliche Qualifikation besitzt.

2) BVerfG, Beschl. v. 28.7.2004 - 1 BvR 159/04 -, NJW 2004 2656, 2653.

3) Vgl. Kleine $=$ Cosack, BRAO, 5. Aufl. 2008, Vor $\ 43$ b, Rdnr. 31

4) Vgl. Henssler, AnwB1. 2009, 1, 7 m. Nachw.

5) Vgl. Bornkamm, in: Köhler/Bornkamm, UWG, 28. Aufl. 2010, \5, Rdnr. 2.113.

6) BVerfG, Beschl. v. 23.7.2001 - 1 BvR 873/00 -, NJW 2001, 2788 $=$ MedR 2001, $569 \mathrm{~m}$. Anm. Rieger.

REZENSIONEN

DOI: 10.1007/s00350-011-2938-8

\section{Handbuch Stammzellenrecht. Ein rechtlicher Praxisleitfaden für Naturwissenschaftler, Ärzte und Juristen.}

Von Timo Faltus. (Hallesche Schriften zum Öffentlichen Recht, Bd. 17), Universitätsverlag Halle-Wittenberg, 279 S., kart., $€ 65,00$

Der Autor, Diplom-Jurist und Diplom-Biologe, ist den Lesern dieser Zeitschrift durch seinen rechtlichen Beitrag zum ,schnell evolvierenden Gebiet der Stammzellenforschung“ (MedR 2008, 544-549) bekannt. Er nennt seine neue Monographie zugleich „Handbuch“, „Praxisleitfaden“ oder auch „Handreichung“ im „Normendschungel“. Freilich soll es nach einer Vorbemerkung „der allgemeinen Information und Bildung, nicht aber der Beratung bei individuellen rechtlichen Anliegen“ dienen. Dagegen sprächen die ,ständigen Veränderungen“. Das Buch bietet kein Sachregister und enthält auch erstaunlicherweise kein Literaturverzeichnis, dafür in der „Danksagung“ den folgenden Hinweis: „Sofern ich hier gemachte Aussagen nicht als Zitate gekennzeichnet habe, obwohl diese Aussage schon von einem anderen vor mir gemacht wurde, so ist dies auch der enormen Anzahl an Veröffentlichungen auf dem Stammzellengebiet und dessen raschen (sic!) Weiterentwicklung geschuldet". Der Autor benennt als Förderer seiner Schrift das ,Translationszentrum für Regenerative Medizin“ in Leipzig.

Prof. Dr. iur. Dr. h.c. Adolf Laufs,

Heidelberg, Deutschland
Zu den Vorzügen des Werkes gehören die naturwissenschaftlichen und medizinischen Aufschlüsse, auch zu neuen Aufbrüchen und Perspektiven der Forschung. Richtigerweise kommt auch immer wieder das Embryonenschutzgesetz zum Aufruf, wobei die Auskünfte allerdings im Ganzen nicht die Qualität des Kommentars von Günther, Taupitz und Kaiser erreichen. Zu wenig Tiefgang und Kontur zeigen die Aussagen zur Kernfrage nach dem Rechtsstatus des Embryos. Mit guten Gründen hält der Autor die Stichtagsregel des Stammzellengesetzes für ,fadenscheinig und rechtlich inkonsistent".

Die normativen Grundlagen bis hin zum europäischen Patentrecht umreißt der Verfasser, um schließlich im letzten, weitaus umfangreichsten Kapitel, im Hauptstück eine ,zelltyp- und tätigkeitsbezogene Betrachtung“ anzustellen: über humane Embryonen und Zellen, „tierliche“ Embryonen und Stammzellen, pflanzliche Stammzellen, chimärische Embryonen und (Stamm-)Zellen, am Ende über die internationale Zusammenarbeit auch im Zeichen des Strafrechts. Wie heikel sich das Thema anlässt, zeigen auch die Darlegungen zur ethischen Beratung und zu den Bemühungen der Fachgesellschaften. Die Nutzung humaner embryonaler Stammzellen ist höchst problematisch, weil diese ,,heute ganz überwiegend (noch) durch die Zerstörung menschlicher Embryonen bereitgestellt werden“. Seit menschliche Embryonen - zunächst im Dienste der Fortpflanzung - verfügbar und in Grenzen auch rechtlich handhabbar geworden sind, seit humane Keimzellen im Labor zu Gebote stehen, eröffnen sich der ausgreifenden Wissenschaft immer weitergehende Möglichkeiten, die fortwährend juristische Grenzfragen aufwerfen und den Rechtsstaat herausfordern, so beispielsweise die Produktion artifizieller Gameten. Der für ein Handbuch eher schmale Band führt den Leser auf diese Felder und mahnt wiederholt zu juristischer Vorsicht. 